\title{
Article \\ Factoring continuous characters defined on subgroups of products of topological groups
}

\author{
Mikhail Tkachenko
}

Department of Mathematics, Universidad Autónoma Metropolitana, Av. San Rafael Atlixco 186, Col. Vicentina, Del. Iztapalapa, Mexico City C.P. 09340, Mexico; mich@xanum.uam.mx

\begin{abstract}
We study factorization properties of continuous homomorphisms defined on subgroups (or submonoids) of products of (para)topological groups (or monoids). A typical result is the following one: Let $D=\prod_{i \in I} D_{i}$ be a product of paratopological groups, $S$ be a dense subgroup of $D$, and $\chi$ a continuous character of $S$. Then one can find a finite set $E \subset I$ and continuous characters $\chi_{i}$ of $D_{i}$, for $i \in E$, such that $\chi=\left(\prod_{i \in E} \chi_{i} \circ p_{i}\right)\left\lceil S\right.$, where $p_{i}: D \rightarrow D_{i}$ is the projection.
\end{abstract}

Keywords: Monoid; Group; Character; Homomorphism; Factorization; Roelcke uniformity

To my colleague and friend M.J. Chasco, with great respect

\section{Introduction}

The present article is a natural continuation of $[18,19]$, where we study continuous homomorphisms of subgroups (submonoids) of products of topological groups (monoids). We establish there that in many cases, a continuous homomorphism $f: S \rightarrow H$ of a submonoid (subgroup) $S$ of a product $D=\prod_{i \in I} D_{i}$ of topological monoids (groups) to a topological monoid (group) $H$ admits a factorization in the form

$$
f=g \circ p_{J} \uparrow S,
$$

where $J$ is a "small" subset of the index set $I, p_{J}: D \rightarrow D_{J}=\prod_{i \in J} D_{i}$ is the projection, and $g: p_{J}(S) \rightarrow H$ is a continuous homomorphism. If one can find a finite (countable) set $J$ for which (1) holds true, we say that $f$ has a finite (countable) type. Most of the results in $[18,19]$ present different conditions on $S$ and/or $H$ under which $f$ has a countable or even finite type. Purely algebraic aspects of this study can be found in [4].

In this article we go further and try to decompose a given continuous homomorphism $f: S \rightarrow H$ into a product of 'coordinate' homomorphisms as explained below.

It follows from the Pontryagin-van Kampen duality theory that every continuous homomorphism of a product $D=\prod_{i \in I} D_{i}$ of compact abelian groups to the circle group $\mathbb{T}$ (called character) has a finite type. Hence, every continuous character of $D$ is a linear combination of finitely many continuous characters, each of which depends on exactly one coordinate. This fact remains valid in a considerably more general situation presented by S. Kaplan in [15]:

Proposition 1. Let $\chi$ be a continuous character of a product $\Pi=\prod_{i \in I} G_{i}$ of (reflexive) topological abelian groups. Then one can find pairwise distinct indices $i_{1}, \ldots, i_{n} \in I$ and continuous characters $\chi_{1}, \ldots, \chi_{n}$ of the respective groups $G_{i_{1}}, \ldots, G_{i_{n}}$ such that the equality

$$
\chi(x)=\prod_{k=1}^{n} \chi_{k}\left(x_{i_{k}}\right)
$$

holds for each $x \in \Pi$.

An analysis of the argument presented in [15] shows that one can drop 'reflexive' in the assumptions of Proposition 1. Hence we can reformulate the conclusion of Proposition 1 by saying that the dual group $\Pi^{\wedge}$ is algebraically isomorphic with $\bigoplus_{i \in I} D_{i}^{\wedge}$, the direct sum of the duals of the factors. Our aim is to extend the conclusion of Proposition 1 to 
considerably wider classes of objects like subgroups or submonoids of Cartesian products of monoids or paratopological groups (see Theorem 2, Corollary 1 and Theorems 4, 5 and $6)$.

An important property of the torus $\mathbb{T}$ is that it is an NSS group, which means that there exists an open neighborhood of the identity in $\mathbb{T}$ containing no nontrivial subgroups. Every Lie group is an NSS group. According to [19, Theorem 3.11], every continuous homomorphism of an arbitrary subgroup of a product of topological monoids to a Lie group has a finite type. This is an essential ingredient in several arguments presented in Section 2.

In Section 3 we complement several results from [19, Section 2] about continuous characters of a dense submonoid $S$ of the $P$-modification of a product $D=\prod_{i \in I} D_{i}$ of topologized monoids. We show in Proposition 3 and Example 3 that if $\varphi: S \rightarrow H$ is a nontrivial continuous homomorphism of $S$ to a topologized monoid of countable pseudocharacter, then the family $\mathcal{J}(\chi)$ of the subsets $J$ of the index set $I$ such that $\varphi$ depend on $J$ is often a filter on $I$, and this filter can have empty intersection, even if $S=D$ and the product $D=\mathbb{Z}(2)^{\omega}$ is a compact metrizable topological group (hence the $P$-modification of $D$ is a discrete group).

\section{Notation and Auxiliary Results}

Let $\mathbb{C}$ be the field of complex numbers with the usual Euclidean topology. The torus $\mathbb{T}$ is identified with the multiplicative subgroup $\{z \in \mathbb{C}:|z|=1\}$ of $\mathbb{C}$.

A semigroup is a nonempty set $S$ with a binary associative operation (called multiplication). A semigroup with identity is called a monoid. Clearly a monoid has a unique identity.

A semigroup $S$ with topology is said to be a semitopological semigroup if multiplication in $S$ separately continuous. This is equivalent to saying that the left and right shifts in $S$ are continuous. If multiplication in $S$ is jointly continuous, we say that $S$ is a topological semigroup. The concept of topological monoid is defined similarly.

Assume that $G$ is a semigroup (monoid, group) with a topology. If the left shifts in $G$ are continuous, then $G$ is called left topological semigroup (monoid, group). If both left and right shifts in $G$ are continuous, then $G$ is said to be a semitopological semigroup (monoid, group). Further, if $G$ is a group and multiplication in $G$ is jointly continuous, we say that $G$ is a paratopological group. A paratopological group with continuous inversion is a topological group.

A topologized monoid (group) is a monoid (group) with an arbitrary topology that may have no relation to multiplication in the monoid (group). We say that a left topological monoid $G$ has open left shifts if for every $x \in G$, the left shift $\lambda_{x}$ of $G$ defined by $y \mapsto x \cdot y$ for each $y \in G$, is an open mapping of $G$ to itself.

A character of an arbitrary monoid $G$ is a (not necessarily continuous) homomorphism of $G$ to the torus $\mathbb{T}$. The continuity of a character, if applies, will always be specified explicitly.

In the sequel we follow notation of Proposition 1 . For every $i \in I$, let $p_{i}$ be the projection of $\Pi$ onto the factor $G_{i}$. Then the conclusion of the proposition is equivalent to saying that $\chi=\prod_{k=1}^{n} \chi_{k} \circ p_{i_{k}}$. It is worth noting that the projections $p_{i}$ are continuous open homomorphisms, so the characters $\chi_{1}, \ldots, \chi_{n}$ are 'automatically' continuous. This assertion follows from the next simple result which shows that for finitely many factors, the conclusion of Proposition 1 remains valid, even if the factors are topologized monoids.

Lemma 1. Let $G=G_{1} \times \cdots \times G_{n}$ be a product of topologized monoids and $\chi$ be a continuous homomorphism of $G$ to a topologized semigroup $K$. Then there exist homomorphisms $\chi_{1}, \ldots, \chi_{n}$ of the respective monoids $G_{1}, \ldots, G_{n}$ to $K$ such that $\chi(x)=\chi_{1}\left(x_{1}\right) \cdots \chi_{n}\left(x_{n}\right)$, for each $x=$ $\left(x_{1}, \ldots, x_{n}\right) \in G$. This representation of $\chi$ is unique and the homomorphisms $\chi_{1}, \ldots, \chi_{n}$ are continuous. 
Proof. For every $k=1, \ldots, n$, let $e_{k}$ be the identity of $G_{k}$ and $p_{k}$ be the projection of $G$ onto the factor $G_{k}$. We define a homomorphism $\chi_{k}$ of $G_{k}$ to $K$ by $\chi_{k}(y)=\chi\left(e_{1}, \ldots, y, \ldots, e_{n}\right)$ for every $y \in G_{k}$, where $y$ stands at the $k$ th position in $\left(e_{1}, \ldots, y, \ldots, e_{n}\right)$. A direct verification shows that $\chi(x)=\chi_{1}\left(x_{1}\right) \cdots \chi_{n}\left(x_{n}\right)$, for each $x=\left(x_{1}, \ldots, x_{n}\right) \in G$.

Let $\psi_{1}, \ldots, \psi_{n}$ be homomorphisms of $G_{1}, \ldots, G_{n}$, respectively, to $K$ satisfying $\chi(x)=$ $\psi_{1}\left(x_{1}\right) \cdots \psi_{n}\left(x_{n}\right)$, for each $x \in G$. We fix an integer $k$ with $1 \leq k \leq n$ and for every $y \in G_{k}$, consider the element $\hat{y}=\left(e_{1}, \ldots, y, \ldots, e_{n}\right) \in G$, where $y$ stands at the $k$ th position in $\hat{y}$. Then $\chi_{k}(y)=\chi(\hat{y})=\psi_{k}(y)$, so $\psi_{k}=\chi_{k}$ for each $k \leq n$ and, hence, the representation $\chi(x)=\chi_{1}\left(x_{1}\right) \cdots \chi_{n}\left(x_{n}\right)$ is unique.

It follows from the continuity of the homomorphism $\chi$ and the equalities $\chi_{k}(y)=$ $\chi\left(e_{1}, \ldots, y, \ldots, e_{n}\right)$, where $1 \leq k \leq n$ and $y \in G_{k}$, that $\chi_{1}, \ldots, \chi_{n}$ are continuous.

Let $X=\prod_{i \in I} X_{i}$ be the Tychonoff product of a family $\left\{X_{i}: i \in I\right\}$ of spaces and $a \in X$ be an arbitrary point. For every $i \in I$, the projection of $X$ to the factor $X_{i}$ is denoted by $p_{i}$. Also, for every $x \in X$, we put

$$
\operatorname{diff}(x, a)=\left\{i \in I: p_{i}(x) \neq p_{i}(a)\right\} .
$$

Then

$$
\Sigma X(a)=\{x \in X:|\operatorname{diff}(x, a)| \leq \omega\}
$$

and

$$
\sigma X(a)=\{x \in X:|\operatorname{diff}(x, a)|<\omega\}
$$

are dense subspaces of $X$ which are called respectively the $\Sigma$-product and $\sigma$-product of the family $\left\{X_{i}: i \in I\right\}$ with center at $a$. If every $X_{i}$ is a monoid (group), we will always choose $a$ to be the identity $e$ of $X$. In the latter case, $\Sigma X(e)$ and $\sigma X(e)$ are dense submonoids (subgroups) of the product monoid (group) $X$ and we shorten $\Sigma X(e)$ and $\sigma X(e)$ to $\Sigma X$ and $\sigma X$, respectively.

Assume that $Z$ is a nonempty subset of the product $X=\prod_{i \in I} X_{i}$ of a family $\left\{X_{i}\right.$ : $i \in I\}$ of sets and $f: Z \rightarrow Y$ is an arbitrary mapping. We say that $f$ depends on $J$, for some $J \subset I$, if the equality $f(x)=f(y)$ holds for all $x, y \in Z$ with $p_{J}(x)=p_{J}(y)$, where $p_{J}: X \rightarrow \prod_{i \in J} X_{i}$ is the projection. It is clear that if $f$ depends on $J$, then there exists a mapping $g$ of $p_{J}(Z)$ to $Y$ satisfying $f=g \circ p_{J}\lceil Z$. Conversely, if there exists such a mapping $g$ of $p_{J}(Z)$ to $Y$, then $f$ depends on $J$.

Definition 1. Assume that $D_{i}$ is a monoid with identity $e_{i}$, where $i \in I$. For a nonempty subset $J$ of $I$, we define a retraction $r_{J}$ of $D=\prod_{i \in I} D_{i}$ by letting

$$
r_{J}(x)_{i}= \begin{cases}x_{i} & \text { if } i \in J ; \\ e_{i} & \text { if } i \in I \backslash J,\end{cases}
$$

for each element $x \in D$. A subset $S$ of $D$ is said to be retractable if $r_{J}(S) \subset S$, for each $J \subset I$. If the inclusion $r_{J}(S) \subset S$ holds for each finite set $J \subset I$, we call $S$ finitely retractable.

The concept of finite retractability will be used in Theorem 5 .

Given a space $X$, we denote by $P X$ the underlying set $X$ with the topology whose base consists of all nonempty $G_{\delta}$-sets in $X$. The space $P X$ is usually referred to as the $P$-modification of $X$. If $X$ is a (left) topological group (monoid), then $P X$ with the same multiplication is also a (left) topological group (monoid).

The family of countable subsets of a given set $I$ is denoted by $[I] \leq \omega$.

\section{Factoring continuous characters}

In this section, we deal with not necessarily Hausdorff objects of Topological Algebra. Since a major part of research articles and books on this subject treat exclusively the Hausdorff case, we need to extend several well-known facts to non-Hausdorff monoids 
and groups. We start with the following result that, informally, goes back to Graev's article [12, pp. 52-53].

Lemma 2. Let $G$ be a topological group with identity e, $N$ be the closure of the singleton $\{e\}$ in $G$ and $\pi: G \rightarrow G / N$ the quotient homomorphism. For every continuous homomorphism $f: G \rightarrow H$ to a Hausdorff topological group $H$, there exists a unique homomorphism $g: G / N \rightarrow H$ satisfying $f=g \circ \pi$ and $g$ is automatically continuous.

Proof. Notice that $N$ is a closed invariant subgroup of $G$, so the quotient topological group $G / N$ is a $T_{1}$-space. Hence $G / N$ is Hausdorff. Denote by $K$ the kernel of $f$. Since $H$ is Hausdorff, $K$ is a closed subgroup of $G$. Hence $\operatorname{ker} \pi=N \subset K=\operatorname{ker} f$. It now follows from [2, Proposition 1.5.10] that there exists a homomorphism $g: G / N \rightarrow H$ satisfying $f=g \circ \pi$. Assume that a homomorphism $\tilde{g}: G / N \rightarrow H$ also satisfies $f=\tilde{g} \circ \pi$. If $y \in G / N$, we take an element $x \in G$ with $\pi(x)=y$. Then $g(y)=g(\pi(x))=f(x)$ and, similarly, $\tilde{g}(y)=\tilde{g}(\pi(x))=f(x)$. Hence $\tilde{g}(y)=g(y)$ for each $y \in G / N$, so $\tilde{g}=g$. As $\pi$ is open and continuous, we conclude that $g$ is continuous.

The pair $(G / N, \pi)$ in Lemma 2 is called the Hausdorff reflection of $G$. Abusing terminology, we usually refer to $G / N$ as the Hausdorff reflection of $G$, thus omitting the quotient homomorphism $\pi$. We also denote $G / N$ by $T_{2}(G)$.

Informally speaking, the following lemma states that the functor of Hausdorff reflection in the category of topological groups and continuous homomorphisms respects arbitrary subgroups.

Lemma 3. Let $G$ be a topological group with identity $e, N$ be the closure of the singleton $\{e\}$ in $G$ and $\pi: G \rightarrow G / N$ the quotient homomorphism. Let also $S$ be an arbitrary subgroup of $G$ and $N_{S}=S \cap N$. Then the quotient group $T_{2}(S)=S / N_{S}$ is topologically isomorphic to the subgroup $\pi(S)$ of $T_{2}(G)=G / N$ and the restriction of $\pi$ to $S$ is an open continuous homomorphism of $S$ onto $\pi(S)$.

Proof. It follows from the definition of $\pi$ that every closed subset $C$ of $G$ satisfies $C=$ $\pi^{-1} \pi(C)$. Therefore, if the subgroup $S$ is closed in $G$ then $N \subset S, S=\pi^{-1} \pi(S)$, and the restriction of $\pi$ to $S$ is an open continuous homomorphism of $S$ onto the subgroup $\pi(S)$ of $G / N$. By the first isomorphism theorem, the groups $\pi(S)$ and $S / N$ are topologically isomorphic.

In the general case, let $K$ be the closure of $S$ in $G$. Then $K$ is a closed subgroup of $G, N \subset K$ and, by the above argument, the groups $T_{2}(K)=K / N$ and $\pi(K) \subset T_{2}(G)$ are topologically isomorphic. Hence it suffices to verify that the group $T_{2}(S)$ is topologically isomorphic to the subgroup $\pi(S)$ of $K / N$. To this end we show that the restriction of $\pi$ to $S$ is an open homomorphism onto the subgroup $\pi(S)$ of $K / N$. Let $U$ be a nonempty open set in $K$ and $V=U \cap S$. Since $K=\pi^{-1} \pi(K)$ and $N \subset K$, the set $U$ satisfies the equality $U=\pi^{-1} \pi(U)$. Hence the set $\pi(U) \cap \pi(S)=\pi(U \cap S)=\pi(V)$ is open in $\pi(S)$. Thus $\pi \uparrow S$ is an open homomorphism of $S$ onto $\pi(S)$ whose kernel is $S \cap N$, so the groups $T_{2}(S)$ and $\pi(S)$ are topologically isomorphic.

Let us recall that the precompact Hausdorff reflection of a given topological group $G$ is a pair $\left(H, \varphi_{G}\right)$, where $H$ is a precompact Hausdorff topological group and $\varphi_{G}: G \rightarrow H$ is a continuous onto homomorphism, such that for every continuous homomorphism $g: G \rightarrow K$ to a Hausdorff precompact topological group $K$, there exists a continuous homomorphism $h: H \rightarrow K$ satisfying $g=h \circ \varphi_{G}$. Every topological group $G$ has a precompact Hausdorff reflection and this reflection is unique up to topological isomorphism [13]. The homomorphism $\varphi_{G}$ is referred to as universal for $G$.

Lemma 4. Let $S$ be a dense subgroup of a topological group $G$ and $\left(H, \varphi_{G}\right)$ be the precompact Hausdorff reflection of $G$. Let also $T=\varphi_{G}(S)$ and $\psi=\varphi_{G}\lceil S$. Then $(T, \psi)$ is the precompact Hausdorff reflection of the group $S$. 
Proof. Since $H$ is a precompact Hausdorff topological group, so is its dense subgroup $T$. Therefore it suffices to verify that the continuous onto homomorphism $\psi: S \rightarrow T$ is universal for $S$. Let $g: S \rightarrow K$ be a continuous homomorphism to a precompact Hausdorff group $K$. The completion of $K$, say, $\varrho K$ is a compact Hausdorff topological group. Hence the group $\varrho K$ is complete. Since $S$ is dense in $G, g$ extends to a continuous homomorphism $g^{*}: G \rightarrow \varrho K$. By the universality of $\varphi_{G}$, there exists a continuous homomorphism $h^{*}: H \rightarrow$ $\varrho K$ such that $g^{*}=h^{*} \circ \varphi_{G}$. Let $h$ be the restriction of $h^{*}$ to $T$. Then $g=g^{*}\left\lceil S=h^{*} \circ \varphi_{G}\lceil S=\right.$ $h^{*} \circ \psi=h \circ \psi$. This proves the universality of $\psi$ for $S$.

A subgroup $S$ of a topological abelian group $G$ is said to be dually embedded in $G$ if every continuous character of $S$ extends to a continuous character of $G$. The next lemma is well known in the special case of Hausdorff topological groups [11, Lemma 2.2].

Lemma 5. Every subgroup $S$ of a precompact topological abelian group $G$ is dually embedded in $G$.

Proof. Let $e$ be the identity of $G$ and $N$ be the closure of the singleton $\{e\}$ in $G$. Let also $p: G \rightarrow G / N$ be the quotient homomorphism. Since $G$ is precompact, the pair $(G / N, p)$ is the precompact Hausdorff reflection of $G$. Let $S$ be a subgroup of $G$. Denote by $K$ the closure of $S$ in $G$. It follows from the definition of $N$ that $N \subset K$ and $K=p^{-1} p(K)$, so $K / N \cong p(K)$ and $(p(K), q)$ is the precompact Hausdorff reflection of $K$, where $q=p \nmid K$. Since $S$ is dense in $K$, Lemma 4 implies that $(q(S), q\lceil S)=(p(S), p\lceil S)$ is the precompact Hausdorff reflection of $S$.

Let $\chi$ be a continuous character of $S$. There exists a continuous character $\lambda$ of the subgroup $T=p(S)$ of the precompact Hausdorff group $G / N$ such that $\chi=\lambda \circ p\lceil S$. By [11, Lemma 2.2], $T$ is dually embedded in the Hausdorff precompact abelian group $G / N$, so $\lambda$ extends to a continuous character $\lambda^{*}$ of $G / N$. Hence $\chi^{*}=\lambda^{*} \circ p$ is an extension of $\chi$ to a continuous character of $G$ and $S$ is dually embedded in the group $G$.

The following fact complements Lemma 5 in the non-abelian case.

Lemma 6. Every dense subgroup $S$ of an arbitrary topological group $G$ is dually embedded in $G$.

Proof. Let $\left(H, \varphi_{G}\right)$ be the precompact Hausdorff reflection of the group $G$. We put $T=$ $\varphi_{G}(S)$ and $\psi=\varphi_{G}\lceil S$. By Lemma 4 , the pair $(T, \psi)$ is the precompact Hausdorff reflection of $S$.

Let $\chi$ be a continuous character of $S$. Then there exists a continuous character $\chi_{T}$ of $T$ such that $\chi=\chi_{T} \circ \psi$. Since the group $H$ is precompact and Hausdorff, it follows from [11, Lemma 2.2] that $T$ is dually embedded in $H$. Hence $\chi_{T}$ extends to a continuous character $\lambda$ of $H$. Then $\chi^{*}=\lambda \circ \varphi_{G}$ is a continuous character of $G$ which extends $\chi$.

Lemma 6 is not valid for closed subgroups of Hausdorff topological groups. In fact, even a compact subgroup of a separable metrizable topological abelian group can fail to be dually embedded [2, Example 9.9.61].

According to Proposition 3.6.12 of [2], a continuous homomorphism of a dense subgroup $S$ of a Hausdorff topological group $G$ to a complete Hausdorff topological group $H$ extends to a continuous homomorphism of $G$ to $H$. Below we generalize this fact by showing that it remains valid for dense subgroups of arbitrary paratopological groups. Our argument makes use of the topological group reflection of a paratopological group (see [16]).

Theorem 1. Let $S$ be a dense subgroup of a paratopological group $G$ and $f: S \rightarrow H$ be a continuous homomorphism of $S$ to a complete Hausdorff topological group $H$. Then $f$ extends to a continuous homomorphism $\mathrm{g}: \mathrm{G} \rightarrow \mathrm{H}$.

Proof. Let $i_{G}: G \rightarrow G_{*}$ be the identity mapping of $G$ onto the topological group reflection $G_{*}$ of $G$. It follows from [16, Theorem 12] that the subgroup $T=i_{G}(S)$ of $G_{*}$ is topologically 
isomorphic to the topological group reflection $S_{*}$ of $S$, so we can identify the groups $T$ and $S_{*}$, algebraically and topologically.

Since $H$ is a topological group, there exists a continuous homomorphism $f_{*}: T \rightarrow H$ satisfying $f=f_{*} \circ i_{G}\left\lceil S\right.$. It follows from the continuity of $i_{G}$ that $T$ is a dense subgroup of $G_{*}$. However, the groups $G_{*}$ and $T$ may fail to be Hausdorff.

To reduce our further argument to the case of Hausdorff groups, we denote by $N$ the closure of the singleton $\left\{e_{G}\right\}$ in $G_{*}$ and consider the quotient homomorphism $\pi: G_{*} \rightarrow$ $G_{*} / N$. Then the quotient group $G_{*} / N$ is the Hausdorff reflection of $G_{*}$. By Lemma 3 , the subgroup $\pi(T)$ of $G_{*} / N$ is the Hausdorff reflection of $T$ and the homomorphism $\varphi=\pi \uparrow T$ of $T$ onto $\pi(T)$ is open and continuous. Since the group $H$ is Hausdorff, Lemma 2 implies the existence of a continuous homomorphism $f^{*}: \pi(T) \rightarrow H$ satisfying the equality $f_{*}=f^{*} \circ \varphi$. Notice that $T$ is dense in $G_{*}$ and $\pi(T)$ is dense in $G_{*} / N$. Therefore, by [2, Corollary 3.6.17], $f_{*}$ extends to a continuous homomorphism $g_{*}: G_{*} / N \rightarrow H$ (we use the completeness of $H$ here).

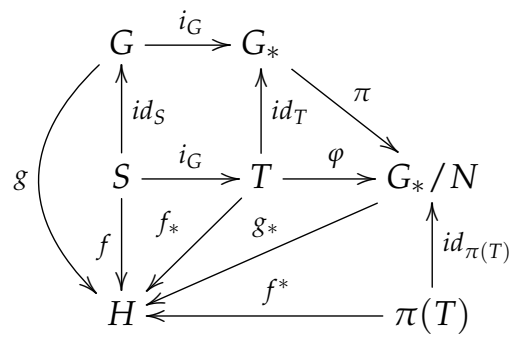

Then $g=g_{*} \circ \pi \circ i_{G}$ is a continuous homomorphism of $G$ to $H$ which extends $f$. This proves the theorem.

We complement Theorem 1 in Proposition 2 by considering continuous homomorphisms defined on dense submonoids of topological monoids.

Example 1. Closed subgroups of completely regular paratopological groups need not be dually embedded. Hence Theorem 1 does not extend to closed subgroups of paratopological groups.

Proof. Let $\mathbb{S}$ be the Sorgenfrey line endowed with the usual topology and addition. Clearly $\mathbb{S}$ is a regular (even hereditarily normal) paratopological group. Let also $\Delta=\{(x,-x): x \in$ $\mathbb{S}\}$ be the second diagonal of $\mathbb{S} \times \mathbb{S}$. It is well known and easy to verify that the subgroup $\Delta$ is discrete and closed. Hence every character of $\Delta$ is continuous and $\Delta$ can be identified with the real line $\mathbb{R}_{d}$ endowed with the discrete topology. On the one hand, an easy calculation shows that the family of characters of $\Delta$ has the cardinality $\mathfrak{c}^{\mathfrak{c}}=2^{\mathfrak{c}}$, where $\mathfrak{c}=2^{\omega}$. On the other hand, the groups $\mathbb{S}$ and $\mathbb{S} \times \mathbb{S}$ are separable, so there are at most $\mathfrak{c}^{\omega}=\mathfrak{c}$ continuous characters of $\mathbb{S} \times \mathbb{S}$. Therefore, not every character of $\Delta$ extends to a continuous character of $\mathbb{S} \times \mathbb{S}$. In other words, $\Delta$ fails to be dually embedded in $\mathbb{S} \times \mathbb{S}$. It is also clear that not every character of $\Delta$ admits the representation described in Lemma 1 (or in Theorem 2 that follows).

The next result is a considerable generalization of Proposition 1.

Theorem 2. Let $D=\prod_{i \in I} D_{i}$ be a product of paratopological groups and $S$ be a subgroup of $D$. Assume that for every finite set $F \subset I$, the subgroup $p_{F}(S)$ of $D_{F}=\prod_{i \in F} D_{i}$ is dually embedded in $D_{F}$, where $p_{F}: D \rightarrow D_{F}$ is the projection. Then for every continuous character $\chi$ of $S$, one can find a finite set $E \subset I$ and continuous characters $\chi_{i}$ of $p_{i}(S)$, for $i \in E$, such that $\chi=\left(\prod_{i \in E} \chi_{i} \circ p_{i}\right)\lceil S$.

Proof. By Corollary 3.12 in [19], one can find a finite set $E \subset I$ and a continuous character $\chi_{E}$ of $p_{E}(S)$ such that $\chi=\chi_{E} \circ p_{E}\left\lceil S\right.$, where $p_{E}: D \rightarrow \prod_{i \in E} D_{i}$ is the projection. By assumptions of the theorem, $T=p_{E}(S)$ is a dually embedded subgroup of $D_{E}=\prod_{i \in E} D_{i}$. 
Hence $\chi_{E}$ extends to a continuous character $\psi$ of $D_{E}$. According to Lemma 1 , for every $i \in E$, there exists a continuous character $\psi_{i}$ of $G_{i}$ such that $\psi=\prod_{i \in E} \psi_{i} \circ q_{i}$, where $q_{i}: D_{E} \rightarrow D_{i}$ is the projection. Let $p_{i}: D \rightarrow D_{i}$ be the projection, for each $i \in E$. Since $p_{i}=q_{i} \circ p_{E}$ and $\chi=\psi \circ p_{E}\left\lceil S\right.$, we conclude that the required equality $\chi=\left(\prod_{i \in E} \chi_{i} \circ p_{i}\right)\lceil S$ is valid.

Example 1 explains why in Theorem 2, we require the projections of a subgroup $S \subset D$ to finite subproducts to be dually embedded, though this does not exclude the possibility that the theorem be valid for arbitrary subgroups of products of (para)topological groups. Later, in Example 2, we will show that such a generalization of Theorem 2 is impossible, even if the factors of the product $D=\prod_{i \in I} D_{i}$ are topological groups.

By Theorem 1, a dense subgroup of a paratopological group is dually embedded. Hence the next corollary is immediate from Theorem 2.

Corollary 1. Let $D=\prod_{i \in I} D_{i}$ be a product of paratopological groups, $S$ be a dense subgroup of $D$, and $\chi$ a continuous character of $S$. Then one can find a finite set $E \subset I$ and continuous characters $\chi_{i}$ of $D_{i}$, for $i \in E$, such that $\chi=\left(\prod_{i \in E} \chi_{i} \circ p_{i}\right) \uparrow S$, where $p_{i}: D \rightarrow D_{i}$ is the projection.

The next example shows that the conditions on $S$ to be 'dually embedded' in Theorem 2 and 'dense' in Corollary 1 are essential.

Example 2. There exist countably infinite, metrizable topological abelian groups $G_{1}, G_{2}$ and a closed discrete subgroup $\Delta$ of the product $\Pi=G_{1} \times G_{2}$ such that $p_{1}(\Delta)=G_{1}, p_{2}(\Delta)=G_{2}$, and the only continuous character of the group $\Pi$ is the trivial one. Here $p_{1}$ and $p_{2}$ are projections of $\Pi$ onto $G_{1}$ and $G_{2}$, respectively. In particular, the trivial character of $\Delta$ is the only one representable in the form described in Corollary 1.

Proof. Let $G$ be a countable, infinite Boolean group. Then $G$ is the direct sum of countably many copies of the group $\mathbb{Z}(2)=\{0,1\}$, so $G$ is as in item 2) of Lemma 0 in [1]. Therefore, Theorem' on page 22 of [1] implies that $G$ admits a metrizable topological group topology $\tau_{1}$ such that the only continuous character of $G_{1}=\left(G, \tau_{1}\right)$ is the trivial one.

Our first observation is that the group $G_{1}$ is not precompact - otherwise continuous characters of $G_{1}$ would separate elements of $G_{1}$. Since every non-zero element of the countable group $G_{1}$ has order 2, one can apply [5, Theorem 5.28] to find an open neighborhood $U$ of zero $e_{1}$ in $G_{1}$ and a (necessarily discontinuous) automorphism $f$ of the group $G_{1}$ such that $f(U) \cap U=\left\{e_{1}\right\}$. In other words, the group $G_{1}$ is self-transversal.

Let $\tau_{2}=\left\{f(V): V \in \tau_{1}\right\}$ be the image of the topology $\tau_{1}$ under the automorphism $f$ and $G_{2}=\left(G, \tau_{2}\right)$. Then $f$ is a topological isomorphism of $G_{1}$ onto $G_{2}$ and the only continuous character of $G_{2}$ is the trivial one. By Lemma 1 the product group $\Pi=G_{1} \times G_{2}$ has the same property. Denote by $\Delta$ the subgroup $\{(x, x): x \in G\}$ of the group $\Pi$. It is clear that $p_{1}(\Delta)=G_{1}$ and $p_{2}(\Delta)=G_{2}$. The set $O=U \times f(U)$ is open in $\Pi$ and it follows from our choice of the set $U$ that the intersection $O \cap \Delta$ contains only the identity element of $G_{1} \times G_{2}$. Hence the subgroup $\Delta$ of $\Pi$ is discrete and closed. It is clear that every character of $\Delta$ is continuous, and that the only character of $\Delta$ that can be expressed in the form presented in Corollary 1 is the trivial one.

Since the subgroup $\Delta$ of the group $G_{1} \times G_{2}$ in Example 2 is discrete, we see that Corollary 1 is not valid for locally compact subgroups of products of topological groups. However, it is valid for precompact abelian subgroups of product groups.

First, we present a well-known result from [10] often called the Comfort-Ross duality for precompact topological abelian groups. We denote the family of all characters of an abstract group $G$ to the torus $\mathbb{T}$ by $\operatorname{Hom}(G, \mathbb{T})$. Clearly, the pointwise multiplication of characters in $\operatorname{Hom}(G, \mathbb{T}),\left(\chi_{1} \cdot \chi_{2}\right)(x)=\chi_{1}(x) \cdot \chi_{2}(x)$, makes it an abelian group.

Theorem 3. For every abelian group $G$, there exists a natural (i.e., functorial) monotone bijection between the family of precompact topological group topologies on $G$ and the subgroups of the group $\operatorname{Hom}(G, \mathbb{T})$. 
'Monotone' in Theorem 3 means that a finer precompact topological group topology on $G$ corresponds to a bigger subgroup of $\operatorname{Hom}(G, \mathbb{T})$. For more details on this correspondence, see [10].

In the following theorem we do not impose any separation restrictions on the factors $D_{i}$ :

Theorem 4. Let $C$ be a precompact abelian subgroup of a product $D=\prod_{i \in I} D_{i}$ of topological groups and $\chi$ be a continuous character of $C$. Then one can find a finite set $E \subset I$ and continuous characters $\chi_{i}$ of $p_{i}(C)$, for $i \in E$, such that $\chi=\left(\prod_{i \in E} \chi_{i} \circ p_{i}\right)\left\lceil C\right.$, where $p_{i}: D \rightarrow D_{i}$ is the projection.

Proof. The projection $p_{i}(C)$ is a precompact abelian subgroup of the group $D_{i}$, for each $i \in I$. We can assume, therefore, that each factor $D_{i}=p_{i}(C)$ is a precompact abelian group. Then $D$ is also a precompact topological abelian group. For every $i \in I$, let $D_{i}^{\wedge}$ be group of continuous characters of $D_{i}$. By [10, Theorem 1.2], the topology of $D_{i}$ is initial with respect to $D_{i}^{\wedge}$. Consider the family

$$
\mathcal{A}=\left\{\chi \circ p_{i}: i \in I, \chi \in D_{i}^{\wedge}\right\}
$$

Then each element of $\mathcal{A}$ is a continuous character of $D$, so $\mathcal{A} \subset D^{\wedge}$. Let $H$ be the subgroup of $D^{\wedge}$ generated by $\mathcal{A}$. Every element $\chi$ of $H$ has the form

$$
\chi=\prod_{k=1}^{n} \chi_{k} \circ p_{i_{k}}
$$

where $i_{1}, \ldots, i_{n}$ are pairwise distinct elements of $I$ and $\chi_{k} \in D_{i_{k}}^{\wedge}$ for each $k=1, \ldots, n$. It is clear that the topology of $D$ is initial with respect to $H$. Since $C$ is a topological subgroup of $D$, the family of restrictions $H_{C}=\{\chi\lceil C: \chi \in H\}$ generates the topology of $C$. Notice that $H_{C}$ is a subgroup of $C^{\wedge} \cap \operatorname{Hom}(C, \mathbb{T})$, so Theorem 3 implies that $H_{C}=C^{\wedge}$. The latter equality together with (3) imply the required conclusion.

Problem 1. Does Theorem 4 extend to precompact subgroups of products of paratopological abelian groups?

The main difficulty in solving Problem 1 is the fact that the topological group reflection of a subgroup $C$ of a paratopological abelian group $D$ can have a strictly finer topology than the topology of $C$ inherited from $D_{*}$. In other words, Lemma 4 cannot be extended to paratopological groups. Even the very special case of Problem 1, where $C$ is a precompact subgroup of the product of two (precompact) paratopological groups, is not clear.

The following result extends a well-known property of continuous homomorphisms of topological groups to a more general case when the domain of a homomorphism is a dense submonoid of a topological monoid with open shifts. First we recall the notions of Roelcke uniformity and Roelcke-completeness in topological groups.

Let $G$ be a topological group and $\mathcal{N}(e)$ be the family of open neighborhoods of the identity $e$ in $G$. For every $U \in \mathcal{N}(e)$, the set

$$
O_{U}=\{U x U: x \in G\}
$$

is an open entourage of the diagonal in $G \times G$ and the family $\left\{O_{U}: U \in \mathcal{N}(e)\right\}$ constitutes a base for a compatible uniformity on $G$, say, $\mathcal{V}_{G}$ which is called the Roelcke uniformity of $G$ (see $\left[2\right.$, Section 1.8]). If the uniform space $\left(G, \mathcal{V}_{G}\right)$ is complete, we say that the group $G$ is Roelcke-complete. 
Proposition 2. Let $S$ be a dense submonoid of a topological monoid D with open shifts. Then every continuous homomorphism $f: S \rightarrow K$ to a Roelcke-complete Hausdorff topological group K extends to a continuous homomorphism $f^{*}: D \rightarrow K$.

Proof. Let $\mathcal{N}(e)$ be the family of open neighborhoods of the identity $e$ in $D$. We denote by $\mathcal{Q}$ the quasi-Roelcke uniformity of $D$ whose base consists of the sets

$$
Q_{V}=\{(x, y) \in D \times D: V x \cap y V \neq \varnothing \neq V y \cap x V\},
$$

where $V \in \mathcal{N}(e)$ (see [3]). It is easy to see that the topology of $D$ generated by $\mathcal{Q}$ is weaker than the original topology of $D$. Let also $\mathcal{V}_{K}$ be the Roelcke uniformity of the group $K$.

Consider a continuous homomorphism $f: S \rightarrow K$ to a Roelcke-complete Hausdorff topological group $K$ with identity $e_{K}$. We claim that $f$ is uniformly continuous considered as a mapping of $\left(S, \mathcal{Q}\lceil S)\right.$ to $\left(K, \mathcal{V}_{K}\right)$. To this end, take an arbitrary symmetric element $U \in \mathcal{N}\left(e_{K}\right)$ and choose an element $W \in \mathcal{N}\left(e_{K}\right)$ such that $W^{2} \subset U$. Then $\bar{W} \subset U$. By the continuity of $f$, we can find an element $V \in \mathcal{N}(e)$ satisfying $f(V \cap S) \subset W$. It remains to verify that $(f(x), f(y)) \in O_{U}$ whenever $(x, y) \in Q_{V} \cap S^{2}$ or, equivalently, $(f \times f)\left(Q_{V} \cap S^{2}\right) \subset O_{U}$.

Let $(x, y) \in Q_{V} \cap S^{2}$. Then $V x \cap y V \neq \varnothing$ and $V y \cap x V \neq \varnothing$. Since $S$ is dense in $D$ and the sets $V x$ and $y V$ are open in $D$, we can choose a point $z \in S \cap V x \cap y V$. It follows from the continuity of shifts in $D$ and the density of $S \cap V$ in $V$ that $z \in V x \subset \overline{(S \cap V) \cdot x}$, the closure is taken in $D$. As $z \in S$, we see that $z$ is in the closure of $(S \cap V) \cdot x$ in $S$. Hence $f(z) \in \overline{f(V \cap S) \cdot f(x)}=\overline{f(V \cap S)} \cdot f(x)$, by the continuity of $f$; the closure is taken in $K$. Since $\overline{f(V \cap S)} \subset \bar{W} \subset U$, the latter implies that $f(z) \in U f(x)$. A similar argument, starting with $z \in y V$, shows that $f(z) \in f(y) U$. Thus $f(z) \in U f(x) \cap f(y) U \neq \varnothing$, whence $f(y) \in U f(x) U^{-1}=U f(x) U$. This implies that $(f(x), f(y)) \in O_{U}$ and proves the uniform continuity of $f$ as a mapping of $\left(S, \mathcal{Q}\lceil S)\right.$ to $\left(K, \mathcal{V}_{K}\right)$.

Since the space $\left(K, \mathcal{V}_{K}\right)$ is complete, $f$ extends to a uniformly continuous mapping $f^{*}:(D, \mathcal{Q}) \rightarrow\left(K, \mathcal{V}_{K}\right)$. It follows from the density of $S$ in $D$ and the Hausdorffness of $K$ that $f^{*}$ is a homomorphism.

Corollary 2. Let $S$ be a dense submonoid of a topological monoid D with open shifts. Then every continuous homomorphism $f: S \rightarrow K$ to a locally compact topological group $K$ extends to a continuous homomorphism $f^{*}: D \rightarrow K$.

Proof. According to Proposition 2 it suffices to verify that every locally compact topological group $K$ is Roelcke-complete. The latter fact is immediate since for every compact neighborhood $U$ of the identity in $K$, every Cauchy filter $\xi$ in the uniform space $\left(K, \mathcal{V}_{K}\right)$ has an element contained in the compact set $U x U$, for some $x \in K$. Hence $\xi$ converges to an element of $K$ and $\left(K, \mathcal{V}_{K}\right)$ is complete, where $\mathcal{V}_{K}$ is the Roelcke uniformity of $K$.

Now we apply Proposition 2 in a less obvious way.

Theorem 5. Let $S$ be a dense submonoid of a product $D=\prod_{i \in I} D_{i}$ of topological monoids with open shifts and $f: S \rightarrow K$ be a continuous homomorphism to a Lie group $K$. If $S$ is either finitely retractable or open in $D$, then $f$ extends to a continuous homomorphism $f^{*}: D \rightarrow K$. Hence, one can find a finite set $E \subset I$ and continuous homomorphisms $\chi_{i}: D_{i} \rightarrow K$ for $i \in E$, such that $f^{*}(x)=\prod_{i \in E} \chi_{i}\left(x_{i}\right)$ for each $x=\left(x_{i}\right)_{i \in I} \in D$.

Proof. Depending on whether $S$ is finitely retractable or open, we apply respectively Theorem 2.12 or Theorem 3.8(b) of [19] to conclude that $f$ depends on a finite set $E \subset I$. In either case, there exists a continuous homomorphism $g: p_{E}(S) \rightarrow K$ satisfying $f=g \circ p_{E}\lceil S$, where $p_{E}$ is the projection of $D$ to $D_{E}=\prod_{i \in E} D_{i}$. Then $p_{E}(S)$ is a dense submonoid of $D_{E}$ and $D_{E}$ is a topological monoid with open shifts, by [19, Lemma 3.5]. So we entitled to apply Proposition 2 to the homomorphism $g$. Hence, there exists a continuous homomorphism 
$g^{*}: D_{E} \rightarrow K$ extending $g$. According to Lemma 1 we can find continuous homomorphisms $\chi_{i}: D_{i} \rightarrow K$ for $i \in E$ such that $g(y)=\prod_{i \in E} \chi_{i}\left(y_{i}\right)$, for each $y=\left(y_{i}\right)_{i \in E}$. Then $f^{*}=g^{*} \circ p_{E}$ is a continuous homomorphism of $D$ to $K$ extending $f$ and satisfying $f^{*}(x)=\prod_{i \in E} \chi_{i}\left(x_{i}\right)$, for each $x \in D$. This implies the required equality for the homomorphism $f$.

According to [19, Theorem 5], every continuous homomorphism $f: S \rightarrow K$ of an arbitrary subgroup $S$ of a product $D$ of topological monoids to a Lie group $K$ has a finite type, i.e., can be represented as the composition of the projection $p_{E}$ of $S$ to a finite subproduct $D_{E}$ of $D$ and a continuous homomorphism of $p_{E}(S)$ to $K$. Therefore, arguing as in the proof of Theorem 5 and applying Proposition 2 we deduce the following:

Theorem 6. Let $D=\prod_{i \in I} D_{i}$ be a product of topological monoids with open shifts, $S$ be a dense subgroup of $D$ and $f: S \rightarrow K$ a continuous homomorphism to a Lie group $K$. Then $f$ extends to a continuous homomorphism $f^{*}: D \rightarrow K$, so one can find a finite set $E \subset I$ and continuous homomorphisms $\chi_{i}: D_{i} \rightarrow K$, for $i \in E$, such that $f^{*}(x)=\prod_{i \in E} \chi_{i}\left(x_{i}\right)$ for each $x=\left(x_{i}\right)_{i \in I} \in D$.

\section{More on continuous homomorphisms of $P$-modifications of products and their dense submonoids}

First we introduce notation which is used in this section and clarifies our aim.

Let $X=\prod_{i \in I} X_{i}$ be the product of a family $\left\{X_{i}: i \in I\right\}$ of sets, $Z$ be a subset of $X$, and $f: Z \rightarrow Y$ be a mapping. Denote by $\mathcal{J}(f)$ the family of all sets $J \subset I$ such that $f$ depends on $J$. Our main concern is to determine the properties of the family $\mathcal{J}(f)$. For example, one can ask whether $\mathcal{J}(f)$ is a filter or whether it has minimal, by inclusion, elements, or even the smallest element. It has been shown by W. Comfort and I. Gotchev in [7-9] that the family $\mathcal{J}(f)$ can have quite a complicated set-theoretic structure, even if $X$ is a Cartesian product of topological spaces and $f$ is a continuous mapping to a space $Y$. It is worth mentioning that the thorough study of the family $\mathcal{J}(f)$ was motivated by a somewhat simpler question on whether $\mathcal{J}(f)$ had a countable element $J \subset I$. The reader can find an extensive bibliography related to this question in the aforementioned articles and in the earlier survey article [14] by M. Hušek.

It turns out that the intersection of the family $\mathcal{J}(f)$, denoted by $J_{f}$, admits a clear description in terms of $f$. We say that an index $i \in I$ is $f$-essential if there exist points $x, y \in Z$ such that $\operatorname{diff}(x, y)=\{i\}$ and $f(x) \neq f(y)$. Let $E_{f}$ be the set of all $f$-essential indices in $I$. By Proposition 2.2 in [17], $J_{f}=E_{f}=\bigcap \mathcal{J}(f)$. In particular, the set $J_{f}$ is empty if and only if no index $i \in I$ is $f$-essential.

Below we present a useful fact which is not valid for arbitrary dense subgroups of the topological group $P D$, the $P$-modification of the product $D=\prod_{i \in I} D_{i}$ of topologized monoids $D_{i}$, not even if the factors $D_{i}$ are finite discrete groups (see [18, Example 1]).

Proposition 3. Let $D=\prod_{i \in I} D_{i}$ be a Cartesian product of topologized monoids, $S$ be a submonoid of $D$ with $\Sigma D \subset S$, and $\varphi: P S \rightarrow H$ a nontrivial continuous homomorphism of the P-modification of $S$ to a topologized monoid $H$ of countable pseudocharacter. Then the family

$$
\mathcal{J}(\varphi)=\{J \subset I: \varphi \text { depends on } J\}
$$

is a filter on the index set $I$.

Proof. Since the subspace $P S$ of $P D$ is a $P$-space, the homomorphism $\varphi: P S \rightarrow P H$ remains continuous (see e.g. [18, Lemma 6]). Notice that $P H$ is a discrete space. Therefore, we can assume that $H$ carries the discrete topology. Applying [18, Proposition 2] we find a countable subset $E$ of $I$ and a continuous homomorphism $\varphi_{E}$ of $p_{E}(S) \subset P D_{E}$ to $H$ such that $\varphi=\varphi_{E} \circ p_{E}\left\lceil S\right.$, where $p_{E}: D \rightarrow D_{E}=\prod_{i \in E} D_{i}$ is the projection. It follows from $\Sigma D \subset S$ that $p_{E}(S)=D_{E}$. Hence $\bar{\varphi}=\varphi_{E} \circ p_{E}$ is a continuous homomorphism of $P D$ to $H$. It follows from the definition of $\bar{\varphi}$ that this homomorphism depends on $E$. Furthermore, if $\bar{\varphi}$ depends on $F$, for some $F \subset I$, then so does $\varphi$. It is now clear that $\mathcal{J}(\bar{\varphi})=\mathcal{J}(\varphi)$. 
Therefore, we can assume without loss of generality that $\varphi$ is a continuous character of $P D=S$. Assume that $J_{1} \subset J_{2} \subset I$ and $J_{1} \in \mathcal{J}(\varphi)$. Then there exists a mapping $g: D_{J_{1}}=\prod_{i \in J_{1}} D_{i} \rightarrow H$ satisfying $\varphi=g \circ p_{J_{1}}$, where $p_{J_{1}}: P D \rightarrow P D_{J_{1}}$ is the projection. Clearly $g$ is a homomorphism. Since the projection $p_{J_{1}}$ is open, the homomorphism $g$ is continuous. Therefore, $g$ is a continuous homomorphism of $P D_{J_{1}}$ to $H$. Let $p_{J_{1}}^{J_{2}}$ be the projection of $D_{J_{2}}$ to $D_{J_{1}}$. Then $\varphi=g \circ p_{J_{1}}=g \circ p_{J_{1}}^{J_{2}} \circ p_{J_{2}}=f \circ p_{J_{2}}$, where $f=g \circ p_{J_{1}}^{J_{2}}$ is a continuous homomorphism of $P D_{J_{2}}$. Hence, $\varphi$ depends on $J_{2}$ and $J_{2} \in \mathcal{J}(\varphi)$.

Let $J_{1}$ and $J_{2}$ be arbitrary elements of $\mathcal{J}(\varphi)$. It is easy to see that $\operatorname{ker} p_{J_{1}} \subset \operatorname{ker} \varphi$ and $\operatorname{ker} p_{J_{2}} \subset \operatorname{ker} \varphi$. Put $J=J_{1} \cap J_{2}$. Then

$$
\operatorname{ker} p_{J}=\operatorname{ker} p_{J_{1}} \cdot \operatorname{ker} p_{J_{2}} \subset \operatorname{ker} \varphi \neq D .
$$

In particular, $J \neq \varnothing$ (we identify $p_{\varnothing}$ with the constant mapping of $D$ to the identity $e_{D}$ of $D$ ). It follows from the inclusion $\operatorname{ker} p_{J} \subset \operatorname{ker} \varphi$ that there exists a homomorphism $h: D_{J} \rightarrow H$ satisfying $\varphi=h \circ p_{J}$ (see [6, Theorem 1.48] or [18, Lemma 2]). We conclude that $J \in \mathcal{J}(\varphi)$.

Summing up, the family $\mathcal{J}(\varphi)$ is a filter.

The reader can find several results about continuous homomorphisms or characters defined on dense submonoids and subgroups of Cartesian (equivalently, Tychonoff) products in [18] and [19]. On many occasions, the conclusions there are stronger than the one in Proposition 3.

It is natural to ask whether the filter $\mathcal{J}(\varphi)$ in Proposition 3 contains a minimal by inclusion element. The next example answers this question in the negative, even if $S$ is the $P$-modification of the compact metrizable group $\mathbb{Z}(2)^{\omega}$ (so $S$ is discrete). Notice that the continuous characters of the compact group $\mathbb{Z}(2)^{\omega}$ are described in Proposition 1.

Example 3. Let the group $G=\mathbb{Z}(2)^{\omega}$ carry the discrete topology. There exist a non-trivial character $\chi$ of $G$ and a decreasing sequence $\left\{J_{n}: n \in \omega\right\}$ of infinite subsets of $\omega$ with empty intersection such that $\chi$ depends on $J_{n}$, for each $n \in \omega$. Hence the filter $\mathcal{J}(\chi)$ does not have minimal elements.

Proof. Let $J_{n}=\omega \backslash\{0,1, \ldots, n\}$, for each $n \in \omega$. Denote by 1 the point of $\mathbb{Z}(2)^{\omega}$ all coordinates of which are equal to 1 . Let also

$$
H_{n}=\left\{x \in \mathbb{Z}(2)^{\omega}: x(i)=0 \text { for each } i \in J_{n}\right\} .
$$

Clearly, $H_{n}$ is a subgroup of $G$ and $H_{n} \subset H_{n+1}$, for each $n \in \omega$. Hence $H=\bigcup_{n=0}^{\infty} H_{n}$ is also a subgroup of $G$. Since $1 \notin H$, there exists a character $\chi$ of $G$ such that $\chi(H)=\{1\}$ and $\chi(\mathbf{1})=-1$. It is immediate from the definition that $\chi$ depends on $J_{n}$, for each $n \in \omega$. Since $\bigcap_{n=0}^{\infty} J_{n}=\varnothing$, the family $\mathcal{J}(\chi)$ has no smallest element. Taking into account that $\mathcal{J}(\chi)$ is a filter (see Proposition 3 ), we infer that it does not contain minimal elements either.

Since the subgroup $H$ of $G$ in the proof of Example 3 is dense in $G=\mathbb{Z}(2)^{\omega}$ provided the latter group is endowed with the usual Tychonoff product topology, the above character $\chi$ is discontinuous on the compact group $\mathbb{Z}(2)^{\omega}$. It turns out that considering the Tychonoff product topology improves the situation greatly - the family $\mathcal{J}(\chi)$ always has a finite minimal (by inclusion) element, for each continuous character $\chi$ of an arbitrary subgroup $G$ of a product of left topological groups. This conclusion can be recovered using techniques from [15] in the special case where $G$ itself is a product of topological groups, but the reader can find a direct argument in the more general [19, Proposition 2.1].

Funding: This research received no external funding.

Conflicts of Interest: The authors declare no conflicts of interest. 


\section{References}

1. Ajtai, M., Havas, I. and Komlós, J. Every group admits a bad topology. Chapter in: Stud. Pure Math. 1983, Springer Basel AG; pp. 21-34.

2. Arhangel'skii, A.V.; Tkachenko, M.G. Topological Groups and Related Structures; van Mill, J., Ed.; Atlantis Press: Paris, France; Amsterdam, The Netherlands, 2008; xiv + 781p, ISBN: 978-90-78677-06-2; doi:10.2991/978-94-91216-35-0_1.

3. Banakh, T. and Ravsky, A. Each regular paratopological group is completely regular. Proc. Amer. Math. Soc. 2017, 145:3, 1373-1382.

4. Bergman, G.M. Homomorphisms on infinite direct products of groups, rings and monoids. Pacific J. Math. 2016, 274, 451-495.

5. Błaszczyk, A. and Tkachenko, A. Transversal, $T_{1}$-independent, and $T_{1}$-complementary topologies. Topol. Appl. 2017, 230, 308-337.

6. Carruth, J.H., Hildebrant, J.A. and Koch, R.J. The Theory of Topological Semigroups, Marcel Dekker, Inc.: New York, NY, USA; Basel, Switzerland, 1983; Volume I.

7. Comfort, W.W. and Gotchev, I. Continuous mappings on subspaces of products with the $\kappa$-box topology. Topol. Appl. 2009, 156, 2600-2608.

8. Comfort, W.W. and Gotchev, I.S. Functional dependence on small sets of indices. Sci. Math. Jpn. 2009, 69, 363-377.

9. Comfort, W.W. and Gotchev, I.S. Cardinal invariants for $\kappa$-box products: weight, density character and Suslin number. Diss. Math. 2016, 516, $41 \mathrm{pp}$.

10. Comfort, W.W. and Ross, K.A. Topologies induced by groups of characters. Fund. Math. 1964, 55, $283-291$.

11. Galindo, J., Tkachenko, M., Bruguera, M., Hernández, C. Reflexivity in precompact groups and extensions. Topol. Appl. 2014, 163 (1), 112-127.

12. Graev, M. I. Theory of topological groups I. Norms and metrics on groups. Complete groups. Free topological groups. Uspekhy Mat. Nauk 1950, 5 (2), 3-56 (in Russian).

13. He, W. and Xiao, Z. The $\tau$-precompact Hausdorff group reflection of topological groups. Bull. Belg. Math. Soc. Simon Stevin 2018, 25, 107-120.

14. Hušek, M. Continuous mappings on subspaces of products. Symp. Math. 1976, 17, 25-41.

15. Kaplan, S. Extension of the Pontrjagin duality I: Infinite products. Duke Math. J. 1948, 15, 649-658.

16. Tkachenko, M. Group reflection and precompact paratopological groups. Topol. Algebra Appl. 2013, 1 , 22-30. doi:102478/taa2013-0003.

17. Tkachenko, M. Factoring continuous mapping defined on subspaces of topological products. Topol. Appl. 2020, 281 , 107198. doi:10.1016/j.topol.2020.107198

18. Tkachenko, M. Factoring continuous homomorphisms defined on submonoids of products of topologized monoids. Axioms 2019, 8, 86. doi:10.3390/axioms8030086.

19. Tkachenko, M. Continuous homomorphisms defined on (dense) submonoids of products of topological monoids. Axioms 2020, 9 , 23. doi:10.3390/axioms 9010023 